Europhys. Lett., 45 (5), pp. 545-551 (1999)

\title{
Coupled Brownian motors: Anomalous hysteresis and zero-bias negative conductance
}

\author{
P. Reimann ${ }^{1}$, R. KaWai ${ }^{2}$, C. Van Den BroecK ${ }^{3}$ and P. HÄngGi ${ }^{1}$ \\ 1 Institut für Physik, Universität Augsburg - D-86135 Augsburg, Germany \\ 2 University of Alabama at Birmingham - Birmingham, AL 35294, USA \\ 3 Limburgs Universitair Centrum - 3590 Diepenbeek, Belgium
}

(received 6 October 1998; accepted in final form 25 December 1998)

PACS. 05.40.-a- Fluctuation phenomena, random processes, noise and Brownian motion.

PACS. 05.60. $+\mathrm{w}-$ Transport processes: theory.

PACS. 64.60.-i - General studies of phase transitions.

\begin{abstract}
We introduce a model of interacting Brownian particles in a symmetric, periodic potential that undergoes a noise-induced non-equilibrium phase transition. The associated spontaneous symmetry breaking entails a ratchet-like transport mechanism. In response to an external force we identify several novel features; among the most prominent being a zero-bias negative conductance and a prima facie counterintuitive, anomalous hysteresis.
\end{abstract}

The subject of this letter lies at the borderline of three topics of current interest. The first - Brownian motors - deals with converting unbiased non-equilibrium fluctuations into useful work, mostly by exploiting the asymmetry of some underlying "ratchet" potential [1]. Proposed as a new paradigm for directed transport in cellular structures and technological applications, their collective behavior, which is our focus here, is clearly of paramount importance [2]. Second, coupled phase oscillators are presently under intense discussion as simple models for the ubiquituous synchronization phenomena in complex biological [3] and physico-chemical systems [4-6]. We will demonstrate here the possibility of spontaneous rotations due to the mere presence of noise and concomitant, quite unexpected response to external forces. Finally, noise-induced phase transitions have been introduced as a spontaneous symmetry breaking caused by non-equilibrium fluctuations in otherwise completely symmetric and monostable systems [7]. This mechanism presents another key ingredient of the present investigation.

Model. - Our starting point is a set of $N$ coupled stochastic equations of motion

$$
\dot{x}_{i}=-U_{i}^{\prime}\left(x_{i}, t\right)+\sqrt{2 T} \xi_{i}(t)-N^{-1} \sum_{j=1}^{N} K\left(x_{i}-x_{j}\right)
$$

in properly scaled units. In doing so we have neglected inertia effects, whereas an important role is played by thermal fluctuations, modeled by the temperature $T$ and the independent $\delta$-correlated Gaussian noises $\xi_{i}(t)$. The last term in (1) is assumed to derive from an interaction 
potential, implying $K(-x)=-K(x)$ (actio=reactio). The potentials $U_{i}(x, t)$ consist of a static part $V(x)$, a fluctuating part $W(x)$, driven by non-thermal noise $\eta_{i}(t)$ with strength $Q$, and possibly an additional bias or "load force" $F$ :

$$
U_{i}(x, t)=V(x)+W(x) \sqrt{2 Q} \eta_{i}(t)-F x .
$$

The potentials $V(x)$ and $W(x)$, as well as the interaction $K(x)$ are supposed to be periodic with period $L$, but unlike in common "ratchets" [1], we furthermore assume symmetric potentials with $V(-x)=V(x)$ and $W(-x)=W(x)$. Our standard example will be

$$
K(x)=K_{0} \sin x \quad, \quad V(x)=W(x)=-\cos x-A \cos 2 x
$$

with $K_{0}>0$ and $L=2 \pi$, corresponding to a "flashing" [1] or "pulsating" potential in (2). Such potential fluctuations may either be externally imposed (in applications), or mimic internal degrees of freedom far from thermal equilibrium (in complex biological systems). For simplicity, we focus on Gaussian white noises $\eta_{i}(t)$ of zero mean and unit strength, independent of each other and all the $\xi_{i}(t)$, and of negligible correlation time (Stratonovich interpretation [8]).

Models of the form $(1,2)$ arise in the context of molecular motors [2], active rotator systems [4], charge density waves [5], planar XY spin models [6], and many others [3]. While for molecular motors [2] a later extension to asymmetric potentials is of prominent interest, in all other contexts [3-6], however, the symmetric case is of foremost interest. The global coupling in (1) and the periodicity of $K(x)$ are chosen to make the problem analytically tractable. We will first discuss in detail this idealized model and afterwards address numerical generalizations for nearest-neighbor coupling (of physical relevance, e.g., in XY spin models), non-periodic $K(x)$, and asymmetric potentials.

The main collective features of $(1,2)$ are captured by the particle density $\rho(x, t):=$ $\sum_{j} \delta\left(x_{j}(t)-x\right) / N$. Two of them are of particular interest, namely the particle current $\langle\dot{x}\rangle:=\partial_{t} \int x \rho(x, t) \mathrm{d} x$ and the reduced density $P(x, t):=\sum_{n} \rho(x+n L, t)$ in the thermodynamic limit $N \rightarrow \infty$. Being an intensive quantity, $P(x, t)$ becomes independent of the specific realization of the noises $\xi_{i}(t)$ and $\eta_{i}(t)$ when $N \rightarrow \infty$ [9]. Equation (1) then yields for the reduced density $P(x, t)$ the non-linear Fokker-Planck equation $[8,9]$

$$
\begin{gathered}
\partial_{t} P(x, t)=\partial_{x}\left\{-f(x, t)+g(x) \partial_{x} g(x)\right\} P(x, t) \\
f(x, t):=-V^{\prime}(x)+F-\int_{-L / 2}^{L / 2} K(x-y) P(y, t) \mathrm{d} y \quad, \quad g(x):=\left[T+Q W^{\prime}(x)^{2}\right]^{1 / 2}
\end{gathered}
$$

with $\int_{-L / 2}^{L / 2} P(x, t) \mathrm{d} x=1$ and $P(x+L, t)=P(x, t)$. For the particle current $\langle\dot{x}\rangle$ one obtains

$$
\langle\dot{x}\rangle=-\int_{-L / 2}^{L / 2} \bar{U}^{\prime}(x) P(x, t) \mathrm{d} x \quad, \quad \bar{U}(x):=V(x)-Q W^{\prime}(x)^{2} / 2-F x .
$$

Spontaneous symmetry breaking. - If $P(x, t)$ is known to approach a steady state $P(x)$ for $t \rightarrow \infty$, then one can infer from (4) that $P(x)$ satisfies the self-consistency equation

$$
P(x)=Z^{-1} \int_{x}^{x+L} \mathrm{~d} y e^{\phi(y)-\phi(x)} /[g(x) g(y)], \quad \phi(x):=-\int_{0}^{x} \mathrm{~d} y f(y) / g(y)^{2},
$$

where $Z$ guarantees $\int_{-L / 2}^{L / 2} P(x) \mathrm{d} x=1$ and $f(y):=f(y, t \rightarrow \infty)$. Equation $(6)$ takes the form

$$
\langle\dot{x}\rangle=L Z^{-1}\left[1-e^{\phi(L)}\right]
$$



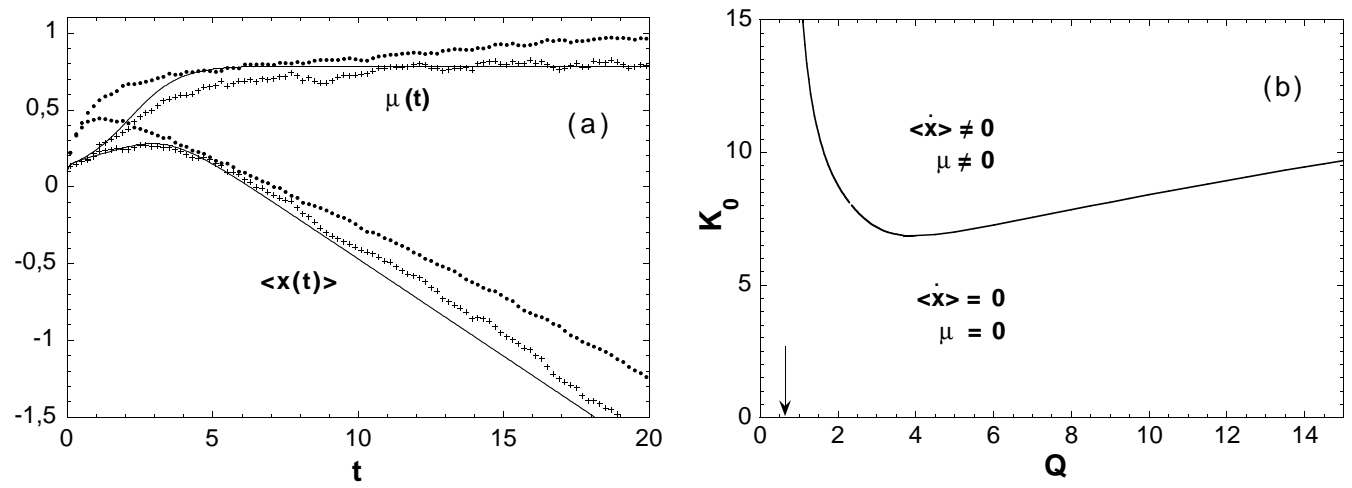

Fig. 1. - (a) Time evolution of $\mu(t)$ from (13) and of $\langle x(t)\rangle:=\int x \rho(x, t) \mathrm{d} x$ for the model (1)-(3) with $T=2, Q=4, F=0, K_{0}=10, A=0.15$, and initial condition $\rho(x, 0)=\delta(x-0.1)$. Solid line: $N \rightarrow \infty$ by solving (4). Crosses: $N=1024$ by simulating (1), averaged over 10 realizations. Dots: simulations with nearest neighbor instead of global coupling in (1) for a $64 \times 64$ square lattice with periodic boundary conditions, averaged over 10 realizations, and modified parameters $Q=10$, $K_{0}=20$. (b) Phase diagram for (1)-(3) with $T=2, F=0, A=0.15$, and $N \rightarrow \infty$. $\langle\dot{x}\rangle$ is the particle current and $\mu$ the order parameter (13) in the steady state $t \rightarrow \infty$. Arrow: asymptotic phase boundary (12) for $K_{0} \rightarrow \infty$.

Note that $\phi(L)$ is decisive for the sign of $\langle\dot{x}\rangle$. Exploiting the fact that $V(x)$ and $W(x)$ are symmetric periodic potentials, and with the definition $\hat{I}:=\int_{-L / 2}^{L / 2} \mathrm{~d} x / g(x)^{2}$ we obtain

$$
\phi(L)=-\hat{I} F-\int_{-L / 2}^{L / 2} \mathrm{~d} x P(x) \int_{-L / 2}^{L / 2} \mathrm{~d} y K(x-y) / g(y)^{2} .
$$

When $F=0$, we conclude that $\phi(L)=0$ and thus $\langle\dot{x}\rangle=0$ if i) $Q=0$ (thermal equilibrium), or ii) $K(x) \equiv 0$, or iii) $P(-x)=P(x)$ (no spontaneously broken symmetry), or iv) $K(x+L / 2)=$ $-K(x)$ and $W^{\prime}(x+L / 2)^{2}=W^{\prime}(x)^{2}$ (accidentally so for (3) if $A=0$ ). In any other generic case, $\langle\dot{x}\rangle \neq 0$ is expected.

Next we discuss the behavior of $P(x, t)$ in (4) for asymptotically strong interactions $K(x)$. To keep things simple, we further assume that multiples of $L$ are the only minima of $V(x)$ and the only zeros of $K(x)$ with $K^{\prime}(x)>0$, cf. (3). As a consequence, all particles in (1) are forced to occupy the same position $\mu(t)$ modulo $L$ and $P(x, t)$ takes the form

$$
P(x, t)=\sum_{n=-\infty}^{\infty} \delta(\mu(t)+n L-x) .
$$

The equation of motion for $\mu(t)$ follows from (4) as

$$
\dot{\mu}(t)=-\bar{U}^{\prime}(\mu(t)) \text {. }
$$

We first focus on the case $F=0$. For small $Q$, the extrema of $\bar{U}(x)$ in (6) are identical to those of $V(x)$. So, $\mu(t)$ in (11) moves (modulo $L$ ) to the minimum $x=0$ of $V(x)$, and $P(x, t)$ approaches a stationary, symmetric limit $P(x)=P(-x)$ for $t \rightarrow \infty$. However, this solution $\mu(t) \equiv 0$ of (11) looses stability and two new stable fixed points appear when $Q$ in (6) exceeds

$$
Q_{\mathrm{c}}:=V^{\prime \prime}(0) / W^{\prime \prime}(0)^{2} .
$$

One thus observes a noise-induced phase transition [7] with a spontaneously broken symmetry of $P(x)$, see fig. 1. Similarly, for a finite (but not too large) bias $F \neq 0$ a stationary $P(x)$ 

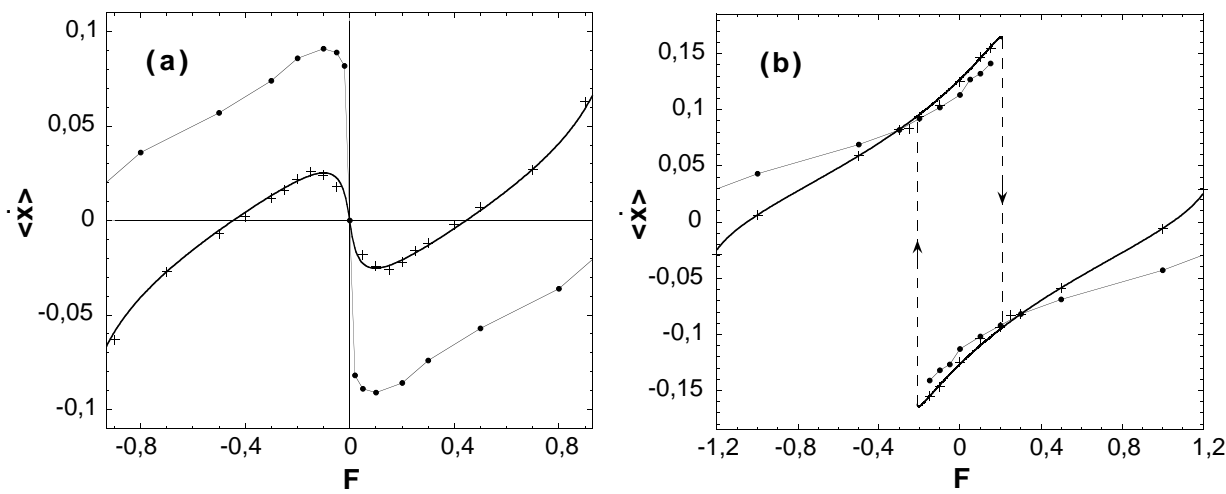

Fig. 2. - (a) Steady-state current vs. force for (1)-(3) with $T=2, Q=2, K_{0}=8, A=0.15$ (global coupling). Solid line: $N \rightarrow \infty$. Crosses: $N=1024$, averaged over 10 realizations. Interconnected dots: $64 \times 64$ square lattice with nearest-neighbor coupling (cf. fig. 1a) and modified parameters $Q=6, K_{0}=15$, averaged over 10 realizations. (b) Same as in (a) but for $Q=4, K_{0}=10$ (global coupling) and $Q=10, K_{0}=20$ (nearest-neighbor coupling).

arises that inherits the broken symmetry of the system and possibly exhibits phase transitions as well. Note that all these asymptotic solutions $P(x)$ are stable (since $\mu(t)$ approaches a local minimum of $\bar{U}(x))$ and further coexisting long-time solutions can be ruled out.

Clearly, many of the above qualitative predictions for $P(x, t)$ will remain valid even for not so strong interactions $K(x)$, see fig. 1. Especially, a stationary, stable long time limit $P(x)$ is expected for not too large tilts $F$, and a suitable generalized definition of $\mu(t)$ is then

$$
\mu(t)=\int_{-L / 2}^{L / 2} x P(x, t) \mathrm{d} x .
$$

For zero bias $F$, a unique, stable, symmetric solution $P(-x)=P(x)$ can be inferred perturbatively from (7) for weak interactions $K(x)$. The very same follows for any finite $K(x)$ and asymptotically large $Q$. Thus for $F=0$ and sufficiently strong interactions, such that at some critical $Q$-value a spontaneous breaking of symmetry does occur, upon further increasing $Q$, the symmetry of $P(x)$ will finally be re-established (re-entrant phase transition [7]). These predictions are confirmed by, and essentially explain the numerical phase diagram in fig. $1 \mathrm{~b}$.

Equipped with these findings for $P(x, t)$, we now turn to their implications for the particle current $\langle\dot{x}\rangle$. As mentioned below (9), for $F=0$ a spontaneous symmetry breaking is necessary - and generically also sufficient- for a corresponding "spontaneous current" $\langle\dot{x}\rangle \neq 0$, see also fig. 1. Roughly speaking, the broken symmetry creates via (5) a periodic but asymmetric effective potential which, out of equilibrium, gives rise to a ratchet-effect [10]. Similarly, for $F \neq 0$ a non-zero current is expected always, apart from certain exceptional parameter combinations, cf. fig. 2. To gain further insight, we focus again on strong interactions $K(x)$. The current (8) is then finite (though small), unless $\phi(L)=0$, and its sign is given by that of $-\phi(L)$. A particularly appealing simplification of (9) is obtained with (3):

$$
\phi(L)=-\hat{I} F-\hat{K} \sin \mu \quad, \quad \hat{K}:=\int_{-L / 2}^{L / 2} \mathrm{~d} x K^{\prime}(x) / g(x)^{2},
$$

where $\mu:=\mu(t \rightarrow \infty)$ follows from (11). Here, a remarkable feature arises, entailing even more striking consequences later on. Namely, if $F=0, Q>Q_{\mathrm{c}}$, and $\hat{K}<0$, which is the case 
whenever $A>0$ in (3), then the sign of $\mu(t)$ from (13) will, in the long time limit, be opposite to that of $\langle\dot{x}\rangle$, see fig. 1a. In other words, for a symmetry broken $P(x)$ with a peak to one side of $x=0$, the flux of particles will move just in the opposite direction!

Anomalous response. - We now come to the response of the steady-state current $\langle\dot{x}\rangle$ to parametric (adiabatically slow) changes of the external force $F$. For $F=0$ and $Q<Q_{\text {c }}$ we have $\langle\dot{x}\rangle=0$, and for strong interactions we can infer from (8), (11), (12), (14) that

$$
\langle\dot{x}\rangle=F L Z^{-1}\left(\hat{I}+\frac{\hat{K} W^{\prime \prime}(0)^{-2}}{Q_{c}-Q}\right)+\mathrm{O}\left(F^{3}\right) .
$$

Thus, for sufficiently large negative $\hat{K}$, a negative zero-bias conductance is predicted. A numerical example for this remarkable behavior is shown in fig. 2a. Apparently, the effect of pulling the particles to one side is analogous to that of a spontaneous symmetry breaking: it generates an effective, coupling-induced "ratchet"-potential in which the non-equilibrium fluctuations promote a current opposite to $F$. Upon approaching the phase boundary, the linear response of $P(x)$ to variations of $F$ diverges, hence the denominator $Q_{\mathrm{c}}-Q$ in (15) and the very steep response curve in fig. 2a. Note that for networks with dead-end loops [11] and in the ratchet work [12], a negative differential conductance (far away from $F=0$ ) has been reported, but not a current opposite to the applied force as in fig. 2a.

For more general $F$ - and $Q$-values and strong interactions (3), the qualitative dependence of $\langle\dot{x}\rangle$ on $F$ follows from (14) by observing how $\mu$ moves in the adiabatically changing potential $\bar{U}(x)$ from (6), see also (11) and discussion thereafter. In this way, not only the continuation of the zero-bias negative conductance beyond $F \simeq 0$ in fig. 2a can be readily understood, but also its even more spectacular counterpart when $Q>Q_{\mathrm{c}}$, namely we discover an anomalous hysteresis loop (fig. 2b) in comparison with the usual hysteresis-cycle as e.g. typified with a ferromagnet. Put differently, given a spontaneous current in one or the other direction, we can apply a small additional force $F$ in the same direction, with the expected result of an increased current in that direction. But upon further increasing $F$, the current will, all of a sudden, switch its direction and run opposite to the applied force! This astonishing feature clearly can only arise for an anomalous hysteresis loop as depicted with fig. $2 \mathrm{~b}$. These response curves in fig. 2 are the result of a competition between the effect of the tilt $F$, favoring a current in that direction, and the ratchet-effect, pumping particles in the opposite direction for $\hat{K}<0$.

Generalizations and summary. - We have studied the effect of a finite (but large) $N$ by comparing direct simulations of (1) (for computational details see [7]) with the $N \rightarrow \infty$ limit following from a straightforward numerical integration of (4). As figs. 1a and 2 illustrate, differences are hardly visible. Note that both numerical schemes (1) and (4) automatically lead to stable long-time limits (with probability one). We have checked this and also excluded further coexisting long-time solutions by starting with different initial conditions both in (1) and (4). While for any finite $N$, occasional jumps between coexisting solutions, e.g. in fig. 2b, are expected theoretically, we have not observed such events within our finite simulation times - a few hundred time-units in (1) - due to the comparatively large $N$-values. An exception is the decay of the metastable state very close to the discontinuities of the hysteresis loop in fig. $2 \mathrm{~b}$.

Next, we have replaced the global coupling in (1) by a nearest-neighbor coupling of the form $\sum_{\langle i j\rangle} K\left(x_{i}-x_{j}\right) / 2 d$ in $d=2$ dimensions by associating the indices $i$ with the vertices of a square lattice with periodic boundary conditions. As figs. 1a and 2 demonstrate, the same qualitative phenomena as for global coupling are recovered, though the quantitative details are of course different. Especially, spontaneous symmetry breaking is again necessary and 
typically also sufficient for the appearance of a spontaneous current, and this, in turn, is a precondition for zero-bias negative conductance and anomalous hysteresis. For $d=1$ neither spontaneous symmetry breaking nor anomalous response has been found.

Further generalizations which we verified numerically are: i) The bare potential $V(x)$ in (2) plays a very minor role: even for $V \equiv 0$ all results remain qualitatively unchanged. Similarly, the thermal noise strength $T$ is arbitrary, except that it must not vanish. ii) In the example (3) with $A=0$, a spontaneous symmetry breaking is possible, but no spontaneous current (see iv) below (9)). This somewhat annoying "accidental symmetry" immediately disappears if in (2) colored noise $\eta_{i}(t)$ is used. iii) A strictly periodic interaction $K(x)$ is not necessary. For instance, one may add on top of the periodic a (not too strong) attractive interaction to keep the "cloud" of particles $x_{i}$ in (1) always well clustered. As long as the symmetric interaction potential, from which $K(x)$ derives, exhibits besides the minimum at $x=0$ at least one appreciable additional pair of (local) minima at $x \approx \pm L$, the same qualitative features as for a strictly periodic $K(x)$ are recovered numerically. A cute application follows by replacing the constant bias $-F x$ in (2) by a slowly varying extra potential term, for instance $-B x^{2}$ with a small $B>0$. In the parameter regime of zero-bias negative conductance, the "cloud" of particles will then spontaneously move towards the maximum at $x=0$ of this potential. Similarly as in i) above, we may even choose $V \equiv 0$ in (2). In this way, deterministically unstable states can thus be stabilized by noise! In the parameter regime of anomalous hysteresis, the "cloud" will permanently oscillate about the potential maximum and so enables one to extract mechanical work solely out of white-noise sources. iv) For asymmetric potentials $V(x)=W(x)$, a similar phase diagram as in fig. 1b arises for $F=0$, but now with both phases exhibiting (the system intrinsic) broken symmetry $\langle\mu\rangle \neq 0$ and a finite particle current $\langle\dot{x}\rangle \neq 0$. The phase boundary thus separates a region with a unique current-carrying stable long-time solution from a region with two coexisting stable long-time solutions. The basic change in the response behavior is a shift of the origin accompanied by an asymmetric deformation of the curves in fig. 2 .

In summary, we have investigated the dynamics of collective Brownian motors where, however, the role of the usually built-in spatial asymmetry of the system is taken over by a spontaneous symmetry breaking due to a noise-induced non-equilibrium phase transition. We note that coupled Brownian motors have been discussed before in the literature [2]. Especially, Jülicher and Prost demonstrated in a somewhat different model of spatially symmetric, globally coupled Brownian motors the generation of a directed current by way of spontaneous symmetry breaking and the closely related features of coexisting phases, first-order transitions, and normal hysteresis. The physics of the class of coupled Brownian motors that we introduced here, however, displays amazing features when exposed to an externally applied force: The reverse hysteresis cycle behavior and the emergence of zero-bias negative conductance are, to our knowledge, completely new collective phenomena. Essentially, they are the result of a competition between the effect of the external bias, favoring current in its direction, and a coupling-induced ratchet potential that arises as a collective property and which pumps particles in the opposite direction.

This work was supported by DFG Sachbeihilfe HA1517/13-2, the Graduiertenkolleg GRK283, NATO grant CRG 950055, the Alabama Education and Research Network, the Alabama Supercomputer Center, the Program on Inter-University Attraction Poles of the Belgian Government, and the F.W.O. Vlaanderen. 


\section{REFERENCES}

[1] Vale R. and Oosawa F., Adv. Biophys., 26 (1990) 97; Ajdari A. and Prost J., C. R. Acad. Sci. Paris, 315 (1992) 1635; Magnasco M., Phys. Rev. Lett., 71 (1993) 1477; Astumian R. and Bier M., Phys. Rev. Lett., 72 (1994) 1766; Doering C., Horsthemke W. and Riordan J., Phys. Rev. Lett., 72 (1994) 2984; Bartussek R., Hänggi P. and Kissner J., Europhys. Lett., 28 (1994) 459.

[2] Derényi I. and Vicsek T., Phys. Rev. Lett., 75 (1995) 374; Jülicher F. and Prost J., Phys. Rev. Lett., 75 (1995) 2618; Marchesoni F., Phys. Rev. Lett., 77 (1996) 2364; Häussler R., Bartussek R. and Hänggi P., in Applied Nonlinear Dynamics and Stochastic Systems near the Millenium, edited by J. Kadtke and A. Bulsara, AIP Proc., 411 (1997) 243; Csahók Z., FAmily F. and Vicsek T., Phys. Rev. E, 55 (1997) 5179; Jülicher F., Ajdari V. and Prost J., Rev. Mod. Phys., 69 (1997) 1269.

[3] Winfree A., The Geometry of Biological Time (Springer, New York) 1980; Kuramoto Y., Chemical Oscillations, Waves, and Turbulence (Springer, New York) 1984; SompolinskY H., Golomb D. and Kleinfeld D., Phys. Rev. A, 43 (1991) 6990; Swift J., Strogatz S. and Wiesenfeld K., Physica D, 55 (1992) 239; Hansel D., Mato G. and Meunier C., Phys. Rev. $E, 48$ (1993) 3470.

[4] Shinomoto S. and Kuramoto Y., Prog. Theor. Phys., 75 (1996) 1105.

[5] Strogatz S., Marcus C., Westervelt R. and Mirollo R., Physica D, 36 (1989) 23.

[6] Arenas A. and Vicente C., Phys. Rev. E, 50 (1994) 949.

[7] Van den Broeck C., Parrondo J. and Toral R., Phys. Rev. Lett., 73 (1994) 3395; Park S. and Kim S., Phys. Rev. E, 53 (1996) 3425; VAn den Broeck C., Parrondo J., Toral R. and Kawai R., ibid., 55 (1997) 4084; Mangioni S., Deza R., Wio H. and Toral R., Phys. Rev. Lett., 79 (1997) 2389; Van den Broeck C. and Kawai R., Phys. Rev. E, 57 (1998) 3866.

[8] Risken H., The Fokker-Planck Equation (Springer, Berlin) 1984.

[9] Desai R. and Zwanzig R., J. Stat. Phys., 19 (1978) 1; Dawson D., J. Stat. Phys., 31 (1983) 29; Bonilla L., J. Stat. Phys., 46 (1987) 659; Strogatz S. and Mirollo R., J. Stat. Phys., 63 (1991) 613.

[10] Reimann P., Phys. Rep., 290 (1997) 149.

[11] Balakrishnan V. and Van Den Broeck C., Physica A, 217 (1995) 1, and references therein.

[12] Cecchi G. and Magnasco M., Phys. Rev. Lett., 76 (1996) 1968. 